\title{
Origins of the Relative Stabilities of Anhydrous and Hydrated D-Mannitol Crystals
}

Teresa M. Dierks and Timothy M. Korter*

Department of Chemistry, Syracuse University, 1-014 Center for Science and Technology, Syracuse, NY 13244-4100, USA

\section{Table of Contents}

Figure S1. Experimental PXRD data for $\alpha, \beta$, and $\delta$ D-mannitol polymorphs compared to previously published X-ray data.

Figure S2. Terahertz spectra collected at $293 \mathrm{~K}$ (red) and $78 \mathrm{~K}$ (blue) from 10 to $90 \mathrm{~cm}^{-1}$ for the anhydrous D-mannitol polymorphs.

Figure S3. Gibbs free energy data calculated for each anhydrous D-mannitol polymorph over the temperature range of $0.0-600.0 \mathrm{~K}$

Table S1. Comparison of optimized D-mannitol crystal structures simulated with 6-311G(d,p) and Ahlrich's VTZ with polarization functions (VTZP).

Table S2. Comparisons of BSSE in $\mathrm{kJ} / \mathrm{mol}$ calculated with $6-311 \mathrm{G}(\mathrm{d}, \mathrm{p})$ and VTZP for the anhydrous D-mannitol polymorphs.

Table S3. Frequencies $\left(\mathrm{cm}^{-1}\right)$ of terahertz spectral peaks for the anhydrous D-mannitol polymorphs and their temperature correlations.

Table S4. Lattice parameters and fractional coordinates of the refined hemihydrate structure.

Table S5. Simulated frequencies and intensities of the refined hemihydrate structure. 

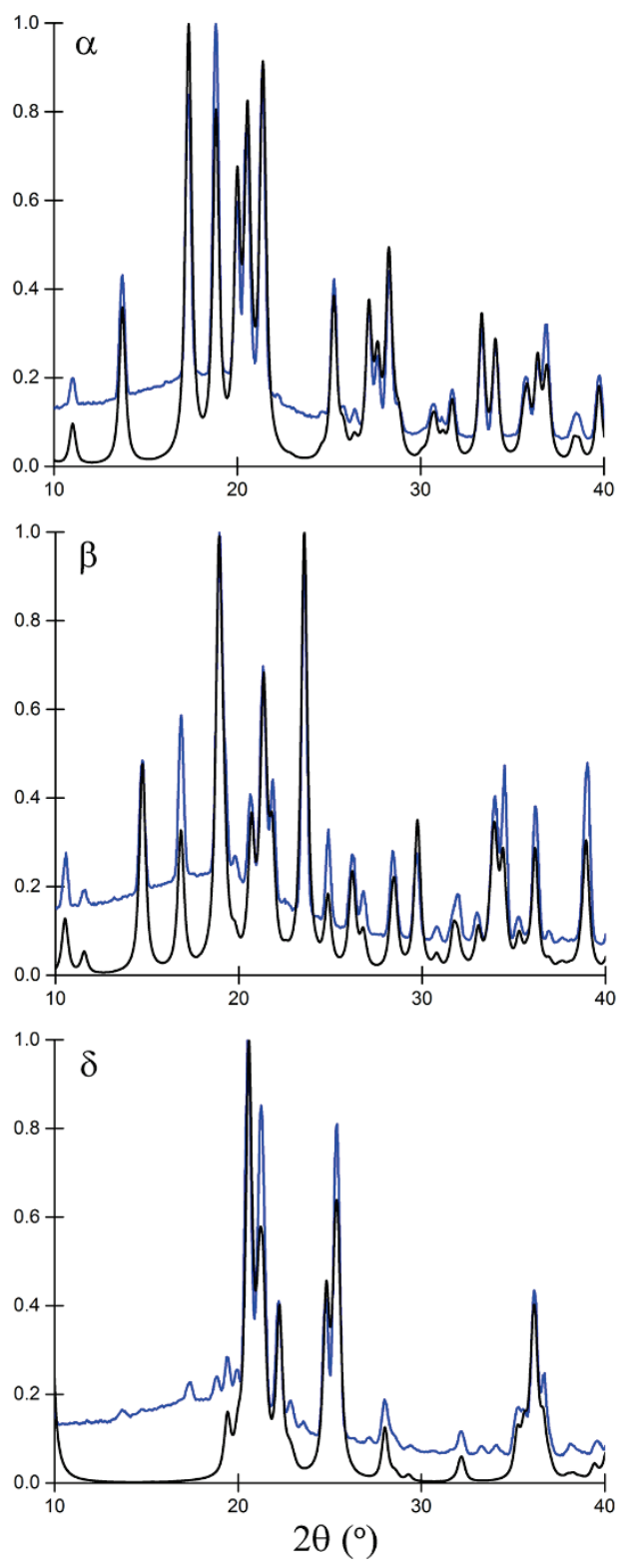

Figure S1. Experimental PXRD data (blue) for $\alpha, \beta$, and $\delta$ mannitol compared to X-ray data published in Fronczek, F.; Kamel, H. N.; Slattery, M. Acta Crystallogr., Sect. C. 2003, 59, 567-570 (black). 


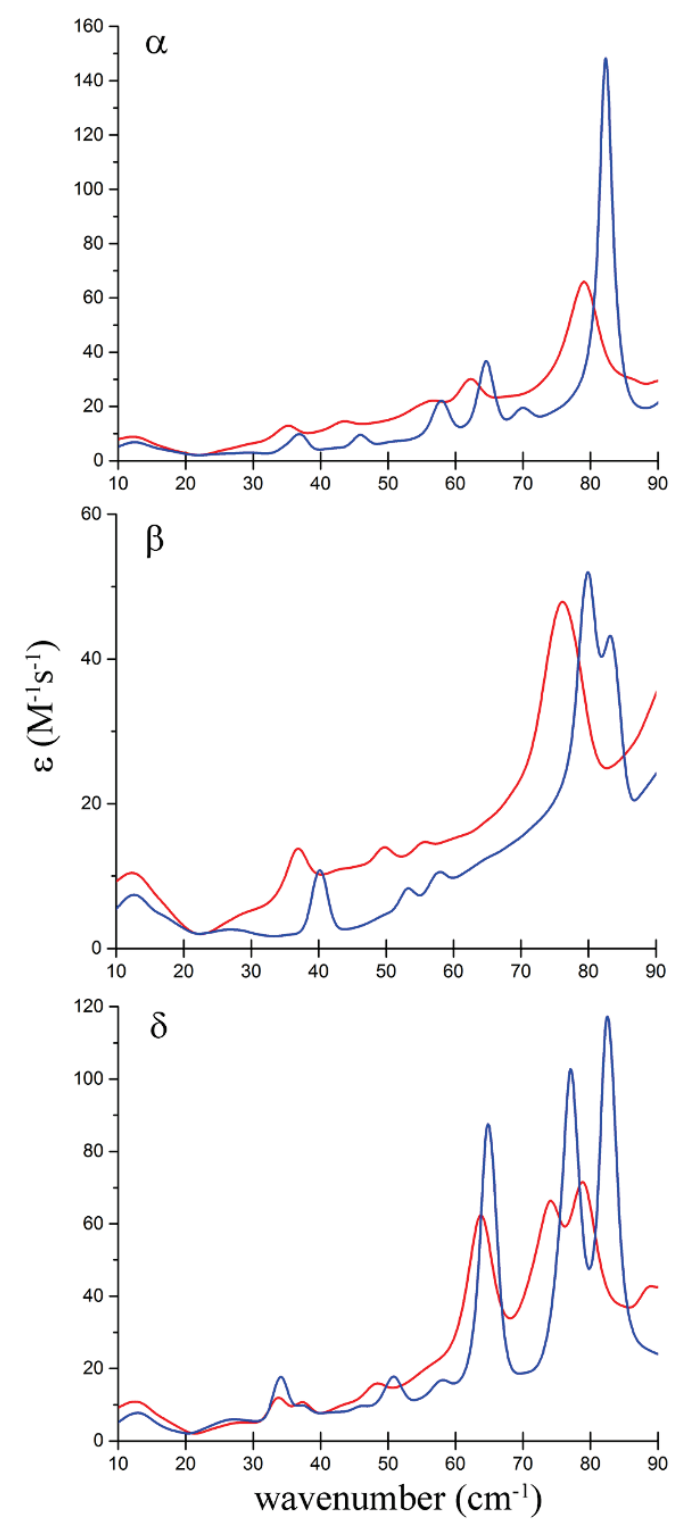

Figure S2. Terahertz spectra collected at $293 \mathrm{~K}$ (red) and $78 \mathrm{~K}$ (blue) from 10 to $90 \mathrm{~cm}^{-1}$ for the anhydrous polymorphs. 

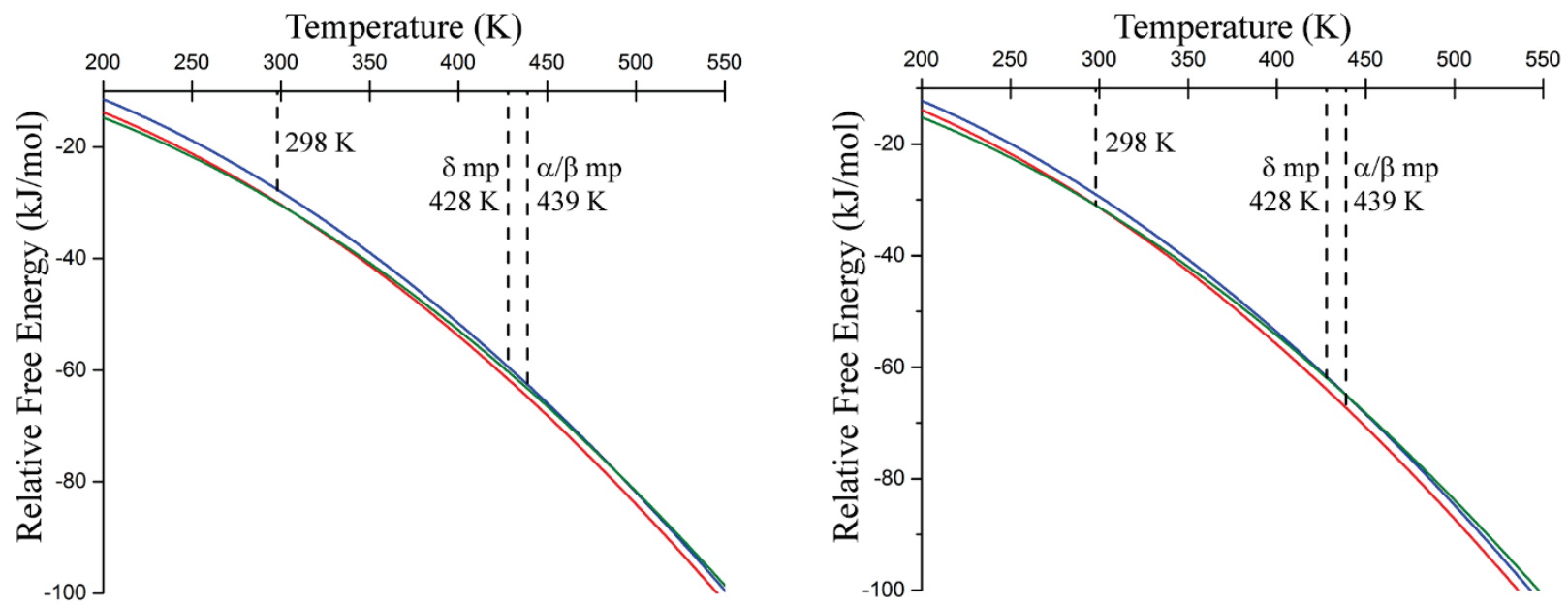

Figure S3. Gibbs free energy data calculated for each anhydrous mannitol polymorph over the temperature range of $0.0-600.0 \mathrm{~K}$. The relative free energy for the fully optimized (left) and constant-volume optimized (right) structures of the $\alpha$ (blue), $\beta$ (red), and $\delta$ (green) polymorphs was plotted over the range of $200.0 \mathrm{~K}-550.0$ to more clearly show the enantiotropic transitions. 
Table S1. Comparison of optimized structures simulated with 6-311G(d,p) and Ahlrich's VTZ with polarization functions (VTZP).

\begin{tabular}{|c|c|c|c|c|c|c|c|c|c|}
\hline & \multicolumn{3}{|c|}{$\alpha$} & \multicolumn{3}{|c|}{$\beta$} & \multicolumn{3}{|c|}{$\boldsymbol{\delta}$} \\
\hline & \multicolumn{9}{|c|}{ 6-311G(d,p) } \\
\hline & Exp. & Calc. & $\%$ Err & Exp. & Calc. & $\%$ Err & Exp. & Calc. & $\%$ Err \\
\hline$a$ & 4.8653 & 4.8280 & -0.77 & 5.5381 & 5.4998 & -0.69 & 4.899 & 4.855 & -0.90 \\
\hline$b$ & 8.873 & 8.671 & -2.27 & 8.580 & 8.369 & -2.46 & 18.268 & 18.109 & -0.87 \\
\hline$c$ & 18.739 & 18.454 & -1.52 & 16.795 & 16.392 & -2.39 & 5.043 & 4.978 & -1.29 \\
\hline \multirow[t]{3}{*}{$\boldsymbol{V}$} & 808.959 & 772.572 & -4.50 & 798.046 & 754.478 & -5.46 & 397.043 & 383.509 & -3.41 \\
\hline & \multicolumn{2}{|c|}{ Abs. Avg. \%Err } & 2.26 & \multicolumn{2}{|c|}{ Abs. Avg. \%Err } & 2.75 & \multicolumn{2}{|c|}{ Abs. Avg. \%Err } & 1.62 \\
\hline & \multicolumn{9}{|c|}{ VTZP } \\
\hline$a$ & 4.8653 & 4.8766 & +0.23 & 5.5381 & 5.5230 & -0.27 & 4.899 & 4.870 & -0.59 \\
\hline$b$ & 8.873 & 8.678 & -2.19 & 8.580 & 8.411 & -1.97 & 18.268 & 18.148 & -0.65 \\
\hline$c$ & 18.739 & 18.556 & -0.97 & 16.795 & 16.547 & -1.48 & 5.043 & 4.999 & -0.86 \\
\hline \multirow[t]{2}{*}{$\boldsymbol{V}$} & 808.959 & 785.321 & -2.92 & 798.046 & 768.681 & -3.68 & 397.043 & 387.617 & -2.37 \\
\hline & \multicolumn{2}{|c|}{ Abs. Avg. \%Err } & 1.58 & Abs. & vg. \%Err & 1.85 & \multicolumn{2}{|c|}{ Abs. Avg. \%Err } & 1.12 \\
\hline
\end{tabular}

Table S2. Comparisons of BSSE in $\mathrm{kJ} / \mathrm{mol}$ calculated with 6-311G(d,p) and VTZP for the anhydrous mannitol polymorphs.

\begin{tabular}{|c|c|c|}
\hline & 6-311G(d,p) & Ahlrich's pVTZ \\
\hline $\boldsymbol{\alpha}$ & 69.17 & 19.43 \\
\hline $\boldsymbol{\beta}$ & 72.75 & 19.73 \\
\hline $\boldsymbol{\delta}$ & 76.34 & 23.24 \\
\hline
\end{tabular}


Table S3. Frequencies $\left(\mathrm{cm}^{-1}\right)$ of terahertz spectral peaks for the anhydrous polymorphs and their temperature correlations.

\begin{tabular}{c|c|c|c|c|c}
\hline \multicolumn{2}{c|}{$\boldsymbol{\alpha}$} & \multicolumn{2}{c|}{$\boldsymbol{\beta}$} & \multicolumn{2}{c}{$\boldsymbol{\delta}$} \\
\hline $\mathbf{2 9 3}$ K & $\mathbf{7 8 ~ K}$ & $\mathbf{2 9 3}$ K & $\mathbf{7 8 ~ K}$ & $\mathbf{2 9 3}$ K & $\mathbf{7 8 ~ K}$ \\
\hline 35.4 & 36.4 & 36.4 & 40.1 & 33.7 & 34.0 \\
43.7 & 45.8 & 50.0 & 53.1 & 37.5 & 38.0 \\
57.3 & 58.3 & 56.2 & 58.3 & 47.9 & 50.7 \\
62.5 & 64.5 & 76.0 & 79.8 & & 56.7 \\
& 69.8 & & 83.4 & 63.6 & 64.9 \\
79.1 & 82.3 & & & 73.6 & 77.0 \\
& & & & 79.1 & 82.7
\end{tabular}


Table S4. Lattice parameters and fractional coordinates of the refined hemihydrate structure

\begin{tabular}{|c|c|c|c|c|c|c|c|}
\hline$a$ & 9.607991 & $\alpha$ & 102.9253 & & & & \\
\hline$b$ & 10.13037 & $\beta$ & 89.4453 & & & & \\
\hline $\mathrm{C}$ & 4.747702 & $\gamma$ & 112.742 & & & & \\
\hline \multirow[t]{2}{*}{ V } & 413.8625 & & & & & & \\
\hline & $X / A$ & $\mathrm{Y} / \mathrm{B}$ & $\mathrm{Z} / \mathrm{C}$ & & $X / A$ & $\mathrm{Y} / \mathrm{B}$ & $\mathrm{Z} / \mathrm{C}$ \\
\hline$C$ & -0.44558 & 0.217386 & 0.13636 & 0 & 0.338103 & -0.45453 & -0.29474 \\
\hline$C$ & -0.31407 & 0.348365 & 0.319701 & 0 & 0.369597 & -0.29881 & 0.247486 \\
\hline$C$ & -0.36693 & 0.47057 & 0.46178 & 0 & 0.143144 & -0.22581 & -0.16281 \\
\hline$C$ & -0.23502 & -0.38492 & -0.39769 & 0 & 0.196049 & -0.06183 & 0.365274 \\
\hline$C$ & -0.28827 & -0.26002 & -0.27806 & $\mathrm{H}$ & 0.220897 & 0.249483 & -0.49923 \\
\hline$C$ & -0.16146 & -0.12118 & -0.10245 & $\mathrm{H}$ & 0.100433 & 0.160563 & 0.175397 \\
\hline 0 & -0.40825 & 0.09227 & 0.028307 & $\mathrm{H}$ & 0.286181 & 0.410009 & 0.166104 \\
\hline 0 & -0.18915 & 0.399062 & 0.152353 & $\mathrm{H}$ & 0.112706 & -0.48955 & -0.35088 \\
\hline 0 & -0.46691 & 0.414952 & -0.33009 & $\mathrm{H}$ & 0.146628 & -0.3459 & 0.284414 \\
\hline 0 & -0.15672 & -0.40904 & -0.17144 & $\mathrm{H}$ & 0.37265 & -0.17488 & -0.18418 \\
\hline 0 & -0.35149 & -0.23451 & 0.476746 & $\mathrm{H}$ & 0.414448 & -0.02526 & 0.470579 \\
\hline 0 & -0.02562 & -0.07366 & -0.24691 & $\mathrm{H}$ & 0.322243 & 0.037721 & -0.23807 \\
\hline $\mathrm{H}$ & -0.47537 & 0.249416 & -0.05164 & $\mathrm{H}$ & 0.110357 & -0.04949 & 0.460858 \\
\hline $\mathrm{H}$ & 0.4568 & 0.187255 & 0.264787 & $\mathrm{H}$ & 0.172159 & -0.16705 & 0.04047 \\
\hline $\mathrm{H}$ & -0.27238 & 0.31434 & 0.492194 & $\mathrm{H}$ & 0.461971 & -0.28899 & 0.355622 \\
\hline $\mathrm{H}$ & -0.42643 & 0.493231 & 0.294559 & $\mathrm{H}$ & 0.339286 & -0.39893 & -0.09511 \\
\hline $\mathrm{H}$ & -0.15859 & -0.35167 & 0.436194 & $\mathrm{H}$ & -0.02223 & 0.384097 & 0.139709 \\
\hline $\mathrm{H}$ & -0.37625 & -0.29528 & -0.13098 & $\mathrm{H}$ & 0.029113 & 0.283438 & -0.27319 \\
\hline $\mathrm{H}$ & -0.13798 & -0.1418 & 0.10453 & 0 & -0.29158 & 0.04947 & -0.49024 \\
\hline $\mathrm{H}$ & -0.19912 & -0.03063 & -0.05561 & $\mathrm{H}$ & -0.18275 & 0.110807 & -0.49002 \\
\hline $\mathrm{H}$ & 0.02997 & -0.13252 & -0.21508 & $\mathrm{H}$ & -0.32389 & 0.079546 & -0.29701 \\
\hline $\mathrm{H}$ & -0.31876 & -0.12799 & 0.492125 & 0 & 0.338103 & -0.45453 & -0.29474 \\
\hline $\mathrm{H}$ & -0.04821 & -0.36242 & -0.18474 & 0 & 0.369597 & -0.29881 & 0.247486 \\
\hline $\mathrm{H}$ & 0.457101 & 0.457794 & -0.31626 & 0 & 0.143144 & -0.22581 & -0.16281 \\
\hline $\mathrm{H}$ & -0.20499 & 0.455814 & 0.022228 & 0 & 0.196049 & -0.06183 & 0.365274 \\
\hline $\mathrm{H}$ & -0.37206 & 0.068332 & 0.195066 & $\mathrm{H}$ & 0.220897 & 0.249483 & -0.49923 \\
\hline$C$ & 0.129383 & 0.251194 & 0.368456 & & & & \\
\hline$C$ & 0.181709 & 0.395876 & 0.272662 & & & & \\
\hline C & 0.212412 & -0.46942 & -0.47379 & & & & \\
\hline$C$ & 0.245015 & -0.32845 & 0.422805 & & & & \\
\hline$C$ & 0.274531 & -0.1936 & -0.32599 & & & & \\
\hline$C$ & 0.308895 & -0.05196 & -0.4259 & & & & \\
\hline 0 & -0.00258 & 0.228923 & -0.47706 & & & & \\
\hline 0 & 0.074765 & 0.390104 & 0.060311 & & & & \\
\hline
\end{tabular}


Table S5. Simulated frequencies and intensities of the refined hemihydrate structure.

\begin{tabular}{|c|c|c|c|c|c|c|c|}
\hline $\begin{array}{l}\text { Frequency } \\
\left(\mathrm{cm}^{-1}\right)\end{array}$ & $\begin{array}{l}\text { Intensity } \\
\text { (km/mol) }\end{array}$ & $\begin{array}{l}\text { Frequency } \\
\left(\mathrm{cm}^{-1}\right)\end{array}$ & $\begin{array}{l}\text { Intensity } \\
\text { (km/mol) }\end{array}$ & $\begin{array}{c}\text { Frequency } \\
\left(\mathrm{cm}^{-1}\right)\end{array}$ & $\begin{array}{l}\text { Intensity } \\
\text { (km/mol) }\end{array}$ & $\begin{array}{l}\text { Frequency } \\
\left(\mathrm{cm}^{-1}\right)\end{array}$ & $\begin{array}{l}\text { Intensity } \\
\text { (km/mol) }\end{array}$ \\
\hline 51.8614 & 0273 & 443.2881 & 0.3213 & 1044.84 & 299.7898 & 1416.459 & 9.9308 \\
\hline 7 & 352 & & 34 & 23 & 92 & 54 & .7377 \\
\hline 68.6 & 167 & 58 & .0986 & 95 & 389.7714 & 465 & 9.683 \\
\hline 87.5031 & 394 & 510.6677 & 35.2258 & 1066.037 & 60.9327 & 429.707 & 46.7466 \\
\hline 93.3217 & & & & & & & 47.7363 \\
\hline 95.5 & 55 & 350 & .041 & 9 & 18 & 95 & 114.4263 \\
\hline 107.8393 & 37 & 02 & 818 & 68 & 2679 & 59.706 & 30.1819 \\
\hline 110. & 39 & & & & 16 & & \\
\hline & & & & & & & \\
\hline & 57 & & 37 & & & & \\
\hline 154.8386 & 3.6694 & 651.2752 & 13.598 & 1120.896 & 53.4826 & 1488.837 & 182.4178 \\
\hline 15 & & 661 & & & 788 & & \\
\hline 164.2936 & 9.3874 & 679.8548 & 14.6892 & 03 & 379 & 458 & 19 \\
\hline 174.837 & 9.8578 & 710.6007 & 376.1607 & .74 & 49.9399 & 1509.352 & 59.5136 \\
\hline 190.4225 & 14.7718 & 63 & 05 & 25 & 904 & 1514.167 & 18.0531 \\
\hline 204.1443 & 82 & 93 & 381 & 83 & 6 & 5.863 & 10 \\
\hline 210.5 & 56 & 41 & 15 & 6 & 96 & 728 & 97 \\
\hline 21 & 3 & 787.4164 & 23 & 3 & 4 & & 72 \\
\hline 221.7064 & 44 & 793.6438 & + & 0 & 2 & 5 & 18 \\
\hline 233.0581 & & & & & & & \\
\hline 244.877 & 17.5505 & 819.8585 & 95.0985 & 272.04 & 33.0031 & & \\
\hline 253.3694 & 1.5909 & 839.5372 & 181.2985 & 1282.604 & 43.4063 & 3055.375 & 35.8852 \\
\hline 259.4349 & 3.4417 & 848.0495 & 363.1264 & 1292.194 & .5925 & 3060.9 & 68.9259 \\
\hline 272.2811 & 23.455 & 858.8875 & 263.1604 & 3.79 & 83.4494 & 3066.973 & 62.2002 \\
\hline 281.2087 & 2.5255 & 883.1215 & 115.7319 & 1.806 & 47.8918 & 3067.853 & 32.9718 \\
\hline 285.9259 & 9.4132 & 883.8534 & 67.44 & 1324.983 & 13.2031 & 3078.588 & 45.8707 \\
\hline 297.4335 & 6.0678 & 900.6123 & 32.7242 & 1336.919 & 29.1824 & 3086.085 & 9.765 \\
\hline 305.4809 & 29.4305 & 905.6623 & 51.3647 & 1353.353 & 28.1532 & 3090.916 & 1.0359 \\
\hline 315.1186 & 21.6872 & 907.5639 & 172.0276 & 1357.096 & 22.471 & 3104.59 & 36.6713 \\
\hline 318.8662 & 4.5683 & 910.2369 & 30.4895 & 1359.384 & 55.9908 & 3108.34 & 29.8047 \\
\hline 330.9939 & 9.0025 & 921.7348 & 365.9481 & 1372.276 & 70.1161 & 3110.422 & 71.473 \\
\hline 346.1055 & 26.3952 & 946.1268 & 31.0256 & 1375.319 & 69.8491 & 3116.963 & 33.0441 \\
\hline 350.3618 & 22.1723 & 959.6773 & 154.6588 & 1380.111 & 56.1862 & 3117.851 & 9.1713 \\
\hline 359.016 & 43.9748 & 968.0354 & 39.7477 & 1391.906 & 6.8571 & 3128.639 & 33.8222 \\
\hline 375.0239 & 4.9137 & 982.1921 & 107.041 & 1393.509 & 7.6416 & 130.64 & 49.5974 \\
\hline 376.5504 & 47.093 & 992.3467 & 72.3904 & 1404.338 & 22.248 & 3141.482 & 130.8691 \\
\hline 435.8809 & 16.9778 & 1022.17 & 293.6652 & 1407.54 & 12.0906 & 3164.291 & 14.7931 \\
\hline
\end{tabular}




\begin{tabular}{|c|c|}
\hline $\begin{array}{c}\text { Frequency } \\
\text { (cm }^{-1} \mathbf{)}\end{array}$ & $\begin{array}{c}\text { Intensity } \\
(\mathbf{k m} / \mathbf{m o l})\end{array}$ \\
\hline 3234.649 & 2168.893 \\
\hline 3273.551 & 3829.841 \\
\hline 3315.059 & 304.1689 \\
\hline 3337.677 & 29.6561 \\
\hline 3367.604 & 3088.877 \\
\hline 3384.464 & 1469.144 \\
\hline 3409.532 & 4102.245 \\
\hline 3417.152 & 471.3177 \\
\hline 3493.629 & 1246.916 \\
\hline 3536.914 & 1401.42 \\
\hline 3539.765 & 147.2816 \\
\hline 3558.501 & 342.3875 \\
\hline 3633.445 & 573.6256 \\
\hline 3671.295 & 1217.803 \\
\hline
\end{tabular}

